CYSTIC FIBROSIS

\title{
Increased morbidity associated with chronic infection by an epidemic Pseudomonas aeruginosa strain in CF patients
}

\author{
M Al-Aloul, J Crawley, C Winstanley, C A Hart, M J Ledson, M J Walshaw
}

Thorax 2004;59:334-336. doi: 10.1136/thx.2003.014258

See end of article for authors' affiliations Correspondence to Dr M J Walshaw, The Cardiothoracic Centre, Thomas Drive, Liverpool L14 3PE, UK; MWalshaw@ doctors.org.uk

Received 5 August 2003 Accepted

24 November 2003

\begin{abstract}
Background: Chronic pulmonary infection with transmissible Pseudomonas aeruginosa strains in individuals with cystic fibrosis (CF) has been reported, raising issues of cross infection and patient segregation. The first such strain to be described (the Liverpool epidemic strain, LES) is now widespread in many UK CF centres. However, whether such infection carries a worse prognosis is unknown. To address this, the clinical course of a group of CF patients chronically infected by LES was compared with that in patients harbouring unique strains.

Methods: Using $P$ aeruginosa strain genotyping, two cohorts of CF patients attending the Liverpool CF service were identified who were LES positive or negative in 1998 and remained so until 2002. From these, two groups of 12 patients were matched in 1998 for age, spirometric parameters, and nutritional state and their clinical course was followed for 5 years. Patients chronically infected with Burkholderia cepacia were excluded.

Results: Patients chronically infected with LES had a greater annual loss of lung function than those not chronically infected by LES (mean difference between groups $-4.4 \%(95 \% \mathrm{Cl}-8.1$ to $-0.9 ; \mathrm{p}<0.02)$ ), and by 2002 their percentage predicted forced expiratory volume in 1 second $\left(\mathrm{FEV}_{1}\right)$ was worse (mean $65.0 \%$ v 82.6\%, $\mathrm{p}<0.03$ ). Their nutritional state also deteriorated over the study period (mean difference between groups in body mass index $-0.7(95 \% \mathrm{Cl}-1.2$ to $-0.2 ; \mathrm{p}<0.01))$, such that by 2002 they were malnourished compared with LES negative patients (mean BMI 19.4 v 22.7, $\mathrm{p}<0.02$ ).

Conclusions: Chronic infection with the Liverpool epidemic $P$ aeruginosa strain in CF patients confers a worse prognosis than infection with unique strains alone, confirming the need for patient segregation. Since this strain is common in many CF units, strain identification in all CF centres is essential. This can only be carried out using genomic typing methods.
\end{abstract}

$\mathrm{H}$ ighly transmissible and often multiresistant strains of Pseudomonas aeruginosa are increasingly being reported in cystic fibrosis (CF) clinics in the UK, ${ }^{1-3}$ prompting calls for segregation of these chronically infected patients from the remainder. The first such strain to be described was found to be prevalent in patients with CF in Liverpool in $1996^{1}$ and has subsequently been shown to be widespread in CF clinics in the UK. ${ }^{4}$ However, whether chronic infection by epidemic strains carries a worse prognosis is unknown. ${ }^{5}$ To investigate this we compared the clinical course over 5 years of two groups of matched CF patients, one chronically infected by the Liverpool epidemic strain (LES) and one harbouring unique $P$ aeruginosa strains.

\section{METHODS}

We regularly genotype isolates of $P$ aeruginosa in our centre. Using this method, in 2002 we identified all 12 patients who were LES negative in 1998 and who had remained so over the following 5 years. During this 5 year period 17 patients chronically infected by LES died but there were no deaths in LES negative patients. Based on 1998 data, we matched these LES negative patients with 12 who were LES positive for age, sex, spirometric parameters, and nutritional state (table 1) and who were still alive in 2002. There was no difference in the incidence of CF related diabetes or liver disease between the two groups, or in the use of rhDNase or maintenance antibiotics (table 1). We ensured that only patients treated at the same paediatric and adult centres were included to eliminate inter-hospital differences in care protocols and possible strain variation due to geographical location. Patients chronically infected by Burkholderia cepacia and those not chronically infected by $P$ aeruginosa (chronic infection defined as three or more positive sputum cultures in a 6 month period) were excluded. On this basis, a total population of about 90 patients was available for study.

Records were kept of the number of admissions with pulmonary exacerbations, outpatient clinic attendances (scheduled and urgent), respiratory exacerbations requiring oral or intravenous antibiotics, and the total number of intravenous antibiotic days. Antibiotic use was cross correlated against hospital pharmacy and central home care records. Paired forced expiratory volume in 1 second $\left(\mathrm{FEV}_{1}\right)$ $\%$ predicted and body mass index (BMI) were recorded at each clinic visit and inpatient stay, and the best (maximum) values obtained during each calendar year were selected for the study.

\section{Statistical analysis}

To analyse possible trends in lung function and nutritional state, regression lines were fitted to the 5 year data of each individual to estimate the change per year and the slopes between those with and without LES were compared using the unpaired Student's $t$ test. Size of effect was expressed as group mean differences with $95 \%$ confidence intervals and the variation in individual groups as SD/SE of the mean. Proportions of patients with diabetes, liver disease, maintenance antibiotic use, azithromycin use, and rhDNase therapy were compared using a $\chi^{2}$ test. The Wilcoxon rank sum test was used to analyse non-parametric data.

\section{RESULTS}

Patients chronically infected with LES had a greater loss of lung function than LES negative patients (mean (SD) change in $\mathrm{FEV}_{1} \%$ predicted per year $-4.8(5.1) \% v-0.4(3.3) \%$, fig 1$)$. 
Table 1 Patient characteristics in 1998

\begin{tabular}{|c|c|c|c|}
\hline & Epidemic strain ( $n=12$ ) & Unique strains $(n=12)$ & $p$ value \\
\hline Age (years)* & $16.6(2.4 ; 13-20)$ & $17.5(3.7 ; 12-25)$ & NS \\
\hline$M: F$ & $5: 7$ & $7: 5$ & NS \\
\hline $\mathrm{FEV}_{1}(\% \text { predicted })^{*}$ & $86.0(14.8 ; 59-110)$ & $85.3(14.6 ; 60-109)$ & NS \\
\hline BMI $\left(\mathrm{kg} / \mathrm{m}^{2}\right)^{*}$ & $21.0(2.8 ; 16.9-27.1)$ & $21.4(3.4 ; 16.2-28.0)$ & NS \\
\hline CFRD (n) & 2 & 1 & NS \\
\hline Liver disease (n) & 1 & 1 & NS \\
\hline rhDNase therapy (n) & 4 & 3 & NS \\
\hline $\begin{array}{l}\text { Maintenance oral } \\
\text { antibiotics (n) }\end{array}$ & 6 & 6 & NS \\
\hline $\begin{array}{l}\text { Maintenance nebulised } \\
\text { antibiotics ( } n \text { ) }\end{array}$ & 10 & 8 & NS \\
\hline
\end{tabular}

The mean difference between groups in the annual rate of change of $\mathrm{FEV}_{1}$ was $-4.4 \%(95 \% \mathrm{CI}-8.1$ to -0.9 ; $\mathrm{p}<0.02)$, such that by 2002 LES positive patients had a worse $\mathrm{FEV}_{1} \%$ predicted (mean (SD) 65.0 (18.6)\% v 82.6 (20.3)\%, p <0.03).

Furthermore, their nutritional state deteriorated over the study period (mean (SD) rate of change of BMI per year -0.3 (0.6) $v+0.3(0.63)$, fig 2). The mean difference between groups in the annual rate of change in BMI was -0.7 (95\% CI -1.2 to $-0.2 ; \mathrm{p}<0.01$ ), such that by the end of the study they were significantly malnourished compared with LES negative patients (mean (SD) BMI 19.4 (2.3) kg/m² 22.7 (3.5) kg/m², $\mathrm{p}<0.02)$. Although the number of outpatient clinic attendances and respiratory exacerbations requiring oral or intravenous antibiotics did not differ between the two groups (table 2), there was a trend to an increased need for hospital admissions $(p=0.07)$ and days of intravenous antibiotic use $(\mathrm{p}=0.09)$ in the LES group.

More LES positive patients were started on azithromycin for its immune modulatory effect $(5 / 12 \vee 0 / 12, \mathrm{p}<0.05)$ during the 5 year period. No patient had any episodes of ABPA or pneumothorax during the 5 year study period or any major interventions (such as PEG or Portacath insertion).

\section{DISCUSSION}

Highly transmissible (epidemic) strains of $P$ aeruginosa have recently been shown to chronically infect patients with $\mathrm{CF} .{ }^{1-3}$ The first of these was described in our local paediatric centre in 1996, ${ }^{1}$ and this LES has now spread to most CF centres in the $\mathrm{UK}^{4}$ prompting calls for patient segregation. However, whether cross infection with these transmissible, often multiresistant, strains contributes to excess morbidity and mortality in CF patients was previously unknown. ${ }^{5}$ While one study suggested that patients chronically infected by a different (less prevalent) epidemic strain underwent more inpatient treatment over l year, ${ }^{6}$ it was unclear whether

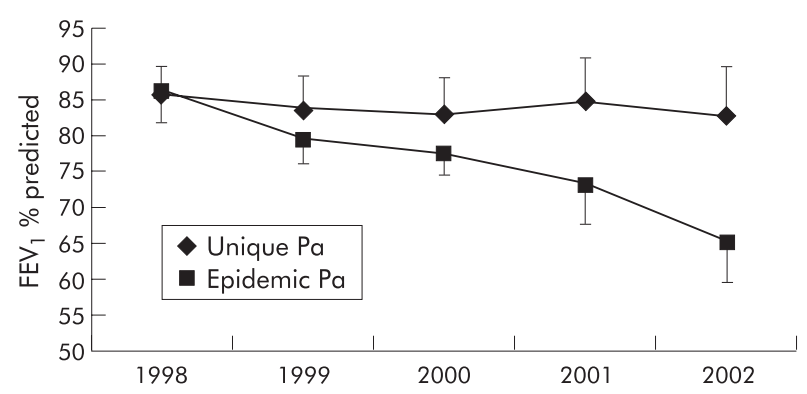

Figure 1 Mean (SE) changes in lung function in CF patients chronically infected with an epidemic strain of $P$ aeruginosa $(\mathrm{Pa})$ and those infected with a unique strain, 1998-2002. $p<0.02$ for difference in the annual rate of change in $\mathrm{FEV}_{1} \%$ predicted.

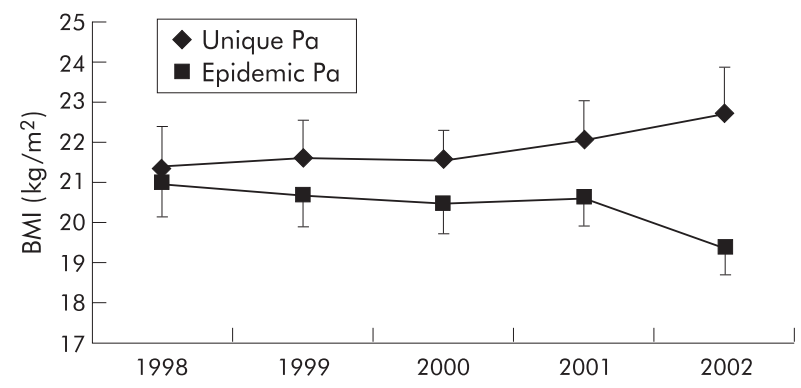

Figure 2 Mean (SE) changes in body mass index (BMI) in CF patients chronically infected with an epidemic strain of $P$ aeruginosa $(\mathrm{Pa})$ and those infected with a unique strain, 1998-2002. $p<0.01$ for difference in the annual rate of change in BMI.

these patients acquired the epidemic strain because they were more severely affected by CF and therefore spent more time in hospital, and not vice versa. Furthermore, this study showed no deterioration in objective parameters of the clinical condition.

For the first time we have clearly shown that a group of $\mathrm{CF}$ patients chronically infected by a major $P$ aeruginosa epidemic strain had a greater deterioration in pulmonary function and nutritional state than matched patients over a prolonged time period. Furthermore, there was a trend to an increased treatment burden in these patients. This is important since this transmissible strain tends to be multiresistant, defined as resistant to all antibiotics in two or more classes of antipseudomonal antibiotics ${ }^{7}$ (as found in 11 of the 12 patients in this study), and we have already shown that the repeated use of the limited range of nephrotoxic antibiotics to which the strain is sensitive has caused renal damage in our adult CF patients. ${ }^{8}$

This study provides compelling evidence that chronic infection by LES is a serious risk factor for CF patients, especially since it is capable of cross infecting patients already harbouring unique strains (superinfection). ${ }^{9}$ However, current segregation policies suggest that all patients chronically infected by $P$ aeruginosa should be separated from the remainder. ${ }^{10}$ Such policies are illogical in that they assume patients infected by unique strains are a risk to the remainder which, by definition, is not possible, ${ }^{11}$ and they also ignore the risk of superinfection by epidemic strains. Furthermore, cohorting all $P$ aeruginosa infected patients together will enhance the contact density, thereby increasing the risk of superinfection. Unfortunately, epidemic strains can only be identified by genomic techniques since antibiogram and morphotype methods are indiscriminatory. ${ }^{212-14}$ In keeping with this, we regularly genotype all $P$ aeruginosa isolates in our unit in order to diminish the risk of cross infection. A rapid 
Table 2 Treatment burden of study patients

\begin{tabular}{lccl}
\hline & Epidemic strain $(\mathbf{n}=12)$ & Unique strains $(\mathbf{n}=12)$ & p value \\
\hline Hospital admissions & $1.6(1.4 ; 0.2-4.4)$ & $0.7(0.5 ; 0.0-1.6)$ & 0.07 \\
$\begin{array}{l}\text { Outpatient clinic visits } \\
\text { Pulmonary exacerbations }\end{array} \quad 4.3(1.3 ; 3.8-8.0)$ & $6.1(1.4 ; 4.0-9.4)$ & 0.51 \\
requiring oral or IV antibiotics & $3.1(1.0 ; 1.4-4.6)$ & 0.24 \\
Total IV antibiotic days $\quad 39.0(26.5 ; 4.4-150.8)$ & $23.1(13.2 ; 0.0-42.2)$ & 0.09 \\
\hline Data are presented as mean (SD; range). & & \\
\hline
\end{tabular}

technique for typing LES directly from sputum has already been described. ${ }^{15}$ Now that LES is common in many other CF units throughout the UK, we advise other CF units to adopt the same procedure.

\section{Authors' affiliations \\ M Al-Aloul, J Crawley, M J Ledson, M J Walshaw, Regional Adult Cystic \\ Fibrosis Unit, The Cardiothoracic Centre, Liverpool, UK \\ C Winstanley, C A Hart, Department of Medical Microbiology, Liverpool University, Liverpool, UK \\ This was an unfunded study. \\ Conflict of interest: none of the authors has declared any conflict of interest. \\ MA collected and analysed the data and wrote the first draft of the paper. JC collected data on antibiotic use and researched central home care records. $\mathrm{CW}$ and $\mathrm{CAH}$ performed the genomic typing of Pseudomonas isolates and provided input into writing the manuscript. MJL contributed ideas to study design and helped write the manuscript. MJW contributed ideas to study design, drafted the manuscript, and supervised the study.}

\section{REFERENCES}

1 Cheng K, Smyth RL, Govan JRW, et al. Spread of $\beta$-lactam-resistant Pseudomonas aeruginosa in a cystic fibrosis clinic. Lancet 1996;348:639-42.

2 Jones AM, Govan JRW, Doherty CJ, et al. Spread of a multiresistant strain of Pseudomonas aeruginosa in an adult cystic fibrosis clinic. Lancet $2001 ; 358: 557-8$
3 Pitt TL. Cross infection of cystic fibrosis patients with Pseudomonas aeruginosa. Thorax 2002;57:921.

4 Scott F, Pitt TL. Identification and characterization of transmissible Pseudomonas aeruginosa strains in UK cystic fibrosis centres. J Cystic Fibrosis 2003:2(Suppl 1):S39.

5 Geddes DM. Of isolates and isolation: Pseudomonas aeruginosa in adults with cystic fibrosis. Lancet 2001;358:522.

6 Jones AM, Dodd ME, Doherty CJ, et al. Increased treatment requirements of patients with cystic fibrosis who harbour a highly transmissible strain of Pseudomonas aeruginosa. Thorax 2002;57:924-5.

7 Cystic Fibrosis Trust Antibiotic Group. Antibiotic treatment for cystic fibrosis. Kent, UK: Cystic Fibrosis Trust, 2002.

8 Al-Aloul M, Govin B, Stockton PA, et al. Antibiotic related renal impairment in adult cystic fibrosis patients. Thorax 2002;57(Suppl III):iii81.

9 McCallum SJ, Corkill J, Gallagher M, et al. Superinfection with a transmissible strain of Pseudomonas aeruginosa in adults with cystic fibrosis chronically colonized by P aeruginosa. Lancet 2001;358:558-60.

10 Cystic Fibrosis Trust Control of Infection Group. Pseudomonas aeruginosa: infection in people with cystic fibrosis: suggestions for prevention and infection control. Kent, UK: Cystic Fibrosis Trust, 2001.

11 Speller DE. Hospital acquired infections. In: Topley \& Wilson's principles of bacteriology. London: Arnold, 1990:142-67.

12 Ojeniyi B, Peterson US, Hoiby N. Comparison of genome fingerprinting with conventional typing methods used on Pseudomonas aeruginosa isolates from cystic fibrosis patients. APMIS 1993;101:168-75.

13 Williams T. Evaluation of antimicrobial sensitivity patterns as markers of Pseudomonas aeruginosa cross infection at a cystic fibrosis clinic. Br J Biomed Sci 1997;54:181-5.

14 Grundmann H, Schneider C, Hartung D, et al. Discriminatory power of three DNA-based typing techniques for Pseudomonas aeruginosa. J Clin Microbiol 1995;33:528-34.

15 Parsons YN, Panagea S, Smart CH, et al. Use of subtractive hybridisation to identify a diagnostic probe for a cystic fibrosis epidemic strain of Pseudomonas aeruginosa. J Clin Microbiol 2002;40:4607-11.

\section{LUNG ALERT}

\section{Ambroxol and reduction of COPD exacerbations}

A Malerba M, Ponticiello A, Radaeli A, et al. Effect of twelve months therapy with oral ambroxol in preventing exacerbations in patients with COPD. Double-blind, randomized, multicenter, placebo-controlled study (the AMETHIST trial). Pulm Pharmacol Ther 2004; 17:27-34

$\mathrm{T}$ his prospective, randomised, double blind, multicentre, placebo controlled study examined the effect of ambroxol (an agent stimulating mucociliary clearance and surfactant production) in preventing acute exacerbations of COPD. 242 patients with GOLD stage IIa COPD, a history of chronic bronchitis, abnormal chest auscultation, and at least one exacerbation in the previous year were recruited and randomised to receive ambroxol or placebo for 12 months. An exacerbation was defined as the presence of purulent sputum with at least one of the following: temperature $>38^{\circ} \mathrm{C}$, general malaise, dyspnoea, difficult expectoration, or new systemic leucocytosis. There was no significant difference in the primary end point-the number of patients free from exacerbationbetween the ambroxol and placebo groups at either 6 or 12 months (63\% v 60\% at 6 months, $56 \%$ v3\% at 12 months, respectively).

Systematic reviews have suggested a significant reduction in the number of exacerbations in patients with mild COPD and chronic bronchitis treated with mucolytic agents. These reviews included many studies using $\mathrm{N}$-acetylcysteine. The AMETHIST trial shows the importance of examining the evidence for individual mucolytic drugs before prescription.

W R Perera

Clinical Research Fellow, St Bartholomew's Hospital, London, UK; Wayomiperera@aol.com 